\title{
Decreased perivascular CGRP-containing nerves in Otsuka Long-Evans Tokushima Fatty rats with insulin resistance and hypertension
}

\begin{abstract}
Shingo Takatori ${ }^{1,2}$, Hiroki Fujiwara ${ }^{2}$, Yoshito Zamami ${ }^{1}$, Narumi Hashikawa-Hobara ${ }^{3}$ and Hiromu Kawasaki ${ }^{1,2}$
We previously reported that chronic hyperinsulinemia induced by drinking fructose elicited an abnormal neuronal regulation of vascular tone, which contributed to the development of hypertension. This study was designed to elucidate the possible mechanisms underlying the dysfunctional neuronal regulation of vascular tone induced by chronic hyperinsulinemia by comparing isolated mesenteric vascular beds from Otsuka Long-Evans Tokushima Fatty (OLETF) rats with those of control Long-Evans Tokushima Otsuka rat (LETO) rats. Insulin, triglyceride and total cholesterol levels in plasma, blood glucose concentrations, a glucose-insulin index, systolic blood pressure and perivascular innervations were assessed using biochemical and immunohistochemical methods. Mesenteric vascular beds isolated from OLETF and LETO rats were perfused with a Krebs solution containing methoxamine, and changes in perfusion pressure in response to periarterial nerve stimulation (PNS) and the perfusion of vasoactive agents were measured. OLETF rats (8-25 weeks old) showed age-related increases in insulin, triglycerides, total cholesterol, blood glucose, the glucose-insulin index (homeostasis model assessment ratio (HOMA-IR)) and systolic blood pressure compared with LETO rats. In perfused mesenteric vascular beds, the PNS-induced adrenergic nervemediated vasoconstrictor responses in OLETF rats were significantly greater than those in LETO rats, whereas the PNS-induced calcitonin gene-related peptide (CGRP) nerve-mediated vasodilator responses in OLETF rats were significantly smaller than those in LETO rats. In immunohistochemical experiments, the density of CGRP-immunopositive nerves in the mesenteric arteries of OLETF rats decreased significantly with age. The present findings suggest that the abnormal innervation of perivascular nerves in mesenteric resistance arteries induced by chronic hyperinsulinemia disturbs the neuronal regulation of vascular tone and may cause hypertension in OLETF rats.
\end{abstract}

Hypertension Research (2014) 37, 398-404; doi:10.1038/hr.2013.151; published online 14 November 2013

Keywords: calcitonin gene-related peptide-containing nerve; insulin resistance; OLETF rat; rat mesenteric artery; vascular responsiveness

\begin{abstract}
INTRODUCTION
Diabetes mellitus is a metabolic disease with several potential complications. ${ }^{1,2}$ Clinical findings indicate that patients with type 2 diabetes mellitus have a higher risk of developing hypertension. ${ }^{3-5}$ Interestingly, reports suggest that the acute or chronic infusion of insulin induces changes in blood pressure levels, suggesting a direct causal relationship between circulating insulin levels and blood pressure.6,7 Moreover, studies in vivo and in vitro have demonstrated that insulin, particularly at high doses, can facilitate atherogenesis. ${ }^{8,9}$ This evidence suggests that insulin resistance per se and the associated components of the insulin resistance syndrome contribute to the development of cardiovascular disease. ${ }^{10,11}$ Thus, insulin has an important role as an endogenous regulator of the cardiovascular system.
\end{abstract}

A previous study demonstrated that chronic hyperinsulinemia in fructose-drinking rats (FDRs) resulted in augmented adrenergic nerve-mediated vasoconstriction and reduced calcitonin gene-related peptide-containing (CGRPergic) nerve-mediated vasodilatation, leading to hypertension. ${ }^{12,13}$ In addition, we reported that FDRs also showed a greater density of adrenergic neuropeptide Y (NPY)immunopositive nerves and a lower density of CGRPergic nerves (CGRP-immunopositive nerves) innervating the mesenteric arteries compared with the control group. ${ }^{14}$

In animal models, both central and peripheral sympathetic neural activities have key roles in the development of hypertension. ${ }^{15}$ Studies in humans also show that changes in sympathetic neuronal control of the circulation have a very large effect on age-related hypertension. ${ }^{16,17}$ Meanwhile, we have reported that the density of

${ }^{1}$ Department of Clinical Pharmacy, College of Pharmaceutical Sciences, Matsuyama University, Matsuyama, Japan; ${ }^{2}$ Department of Clinical Pharmaceutical Science, Graduate School of Medicine, Dentistry and Pharmaceutical Sciences, Okayama University, Okayama, Japan and ${ }^{3}$ Department of Life Science, Okayama University of Science, Okayama, Japan

Correspondence: Professor, H Kawasaki, Department of Clinical Pharmacy, College of Pharmaceutical Sciences, Matsuyama University, 4-2 Bunkyo-cho, Matsuyama, Ehime 790-8578, Japan. 
CGRPergic vasodilator nerves in rat mesenteric resistance arteries contributes to the development and maintenance of hypertension in spontaneously hypertensive rats. ${ }^{18-21}$ On the basis of these findings, the hyperinsulinemia induced by insulin resistance is thought to gradually elicit an abnormal neuronal regulation of vascular tone, which may partly contribute to the development of hypertension. Nonetheless, very little is known about the biological actions or neural effects of hyperinsulinemia and/or insulin resistance in type 2 diabetic Otsuka Long-Evans Tokushima Fatty (OLETF) rats.

OLETF rats have been shown to manifest a stable diabetic state that mimics human type 2 diabetes: (1) increasing body weight just after weaning; (2) a late onset of hyperglycemia after 18 weeks of age and diagnosable diabetes after 24 weeks of age; (3) hyperinsulinemia at 24 weeks of age and hyperglycemia associated with decreased plasma insulin after 55 weeks of age. ${ }^{22}$ However, no or few studies have investigated whether the function of perivascular nerves in the mesenteric arteries of OLETF rats changes with aging. Therefore, we examined OLETF rats at 8 and 25 weeks of age, which are the preclinical and just before onset stages of diabetes during which rats exhibit hyperinsulinemia and/or insulin resistance associated with cardiovascular dysfunction.

Thus, in the present study, to clarify the mechanism underlying the dysfunctional neuronal regulation of vascular tone in chronic hyperinsulinemia and/or insulin resistance, changes in the adrenergic and CGRPergic vascular responses to periarterial nerve stimulation (PNS) were investigated in perfused mesenteric vascular beds of OLETF rats and compared with those in age-matched control LongEvans Tokushima Otsuka (LETO; genetic control for the OLETF rats) rats. In addition, the distribution of sympathetic adrenergic nerves and CGRPergic nerves in the mesenteric arteries of OLETF and LETO rats was examined using immunohistochemical techniques.

\section{METHODS}

\section{Animals}

Six-week-old OLETF rats and age-matched LETO rats were supplied by Otsuka Pharmaceutical (Tokushima, Japan). The animals were given food and water ad libitum. They were housed in the Animal Research Center of Okayama University at a controlled ambient temperature of $22 \pm 2{ }^{\circ} \mathrm{C}$ with $50 \pm 10 \%$ relative humidity and a 12 -h light/12-h dark cycle (lights on at $0800 \mathrm{~h}$ ). Body weight and food and liquid intake were measured at 8 and 25 weeks of age. All experimental procedures were conducted according to the guidelines for Animal Experiments at Okayama University Advanced Science Research Center, Japanese Government Animal Protection and Management Law (No. $105)$ and the Japanese Government Notification on the Feeding and Safekeeping of Animals (No. 6). Every effort was made to minimize the number of animals used and their suffering from pain and stress at each stage of the experiment.

\section{Biochemical analysis}

Under light ether anesthesia, blood samples were obtained by cardiopuncture after a 12-h fast at 8 and 25 weeks of age. Blood glucose levels were immediately measured using a glucose analyzer (ADVANTAGE; Boehringer Mannheim, Tokyo, Japan). The blood samples were then centrifuged to obtain plasma samples, which were stored at $-80^{\circ} \mathrm{C}$. The level of insulin in plasma was determined by a double-antibody method using a solid-phase insulin monoclonal antibody and a guinea-pig insulin antibody with an ELISA insulin kit (Morinaga Biochemistry, Kanagawa, Japan). Total cholesterol and triglyceride levels in plasma were enzymatically determined using commercially available kits (Wako Pure Chemical Industries, , Osaka, Japan). The homeostasis model assessment ratio (HOMA-IR), an index of insulin resistance, was calculated using the formula plasma glucose $\left(\mathrm{mg} \mathrm{dl}^{-1}\right) \times$ insulin $\left(\mu \mathrm{U} \mathrm{ml}^{-1}\right) / 405$.

\section{Measurement of systolic blood pressure and heart rate}

Systolic blood pressure and heart rate were measured in conscious rats by tailcuff plethysmography (TK-370C, Unicom, Tokyo, Japan) between 0900 and $1200 \mathrm{~h}$ at 8 and 25 weeks of age. The average of five readings per animal was used.

\section{Perfusion of rat mesenteric vascular beds}

OLETF and LETO rats were anesthetized with pentobarbital-Na $\left(50 \mathrm{mg} \mathrm{kg}^{-1}\right.$, intraperitoneally), and mesenteric vascular beds were isolated and prepared for perfusion as described previously. ${ }^{23}$ After removal of the entire intestine and associated vascular bed, the mesenteric vascular bed was separated from the intestine by cutting close to the intestinal wall. Only four main arterial branches from the superior mesenteric trunk running to the terminal ileum were perfused. All other branches of the superior mesenteric artery were tied off. The isolated mesenteric vascular bed was then placed in a water-jacketed organ bath maintained at $37^{\circ} \mathrm{C}$ and perfused with a modified (see below) Krebs solution at a constant flow rate of $5 \mathrm{ml} \mathrm{min}^{-1}$ with a peristaltic pump (model AC-2120; ATTO, Tokyo, Japan). The preparation was also superfused with the same solution at a rate of $0.5 \mathrm{ml} \mathrm{min}{ }^{-1}$ to prevent drying. The Krebs solution was bubbled with a mixture of $95 \% \mathrm{O}_{2}-5 \% \mathrm{CO}_{2}$ before passage through a warming coil maintained at $37^{\circ} \mathrm{C}$. The modified Krebs solution was of the following composition (mM): $\mathrm{NaCl} 119.0, \mathrm{KCl} 4.7, \mathrm{CaCl}_{2} 2.4, \mathrm{MgSO}_{4}$ $1.2, \mathrm{NaHCO}_{3} 25.0, \mathrm{KH}_{2} \mathrm{PO}_{4} 1.2$, disodium EDTA 0.03 and dextrose $11.1(\mathrm{pH}$ 7.4). Changes in perfusion pressure were measured with a pressure transducer (model TP-400T; Nihon Kohden, Tokyo, Japan) and recorded using a pen recorder (model U-228; Nippon Denshi Kagaku, Tokyo, Japan).

Experimental protocol for the measurement of vascular responses After 30 min of perfusion, isolated mesenteric vascular beds with a resting tone were subjected to PNS $(4,8$ and $12 \mathrm{~Hz})$ and injections of noradrenaline (5 and $10 \mathrm{nmol}$ ). Thereafter, the Krebs solution was switched to a Krebs solution containing $5 \mu \mathrm{m}$ guanethidine and $7 \mu \mathrm{m}$ methoxamine to increase the perfusion pressure to approximately $100 \mathrm{~mm} \mathrm{Hg}$. Preparations with a methoxamineinduced increase in perfusion pressure $<30 \mathrm{~mm} \mathrm{Hg}$ were excluded from the data analysis. After the perfusion pressure stabilized, PNS (2, 4 and $8 \mathrm{~Hz})$ was performed and injections of rat CGRP (25, 50 and $100 \mathrm{pmol})$, acetylcholine (ACh; 10, 50 and $100 \mathrm{pmol}$ ) and sodium nitroprusside (SNP; 100 and $1000 \mathrm{pmol}$ ) were given to observe changes in perfusion pressure. PNS was performed using bipolar platinum ring electrodes placed around the superior mesenteric artery. Rectangular pulses of $1 \mathrm{~ms}$ and a supramaximal voltage $(50 \mathrm{~V})$ were applied for $30 \mathrm{~s}$ via an electronic stimulator (model SEN 3301; Nihon Kohden, Tokyo, Japan). Noradrenaline, CGRP, ACh and SNP were directly injected into the perfusate proximal to the arterial cannula with an infusion pump (model 975; Harvard Apparatus, Holliston, MA, USA). A volume of $100 \mu \mathrm{l}$ was injected over a period of $12 \mathrm{~s}$.

At the end of each experiment, $100 \mu \mathrm{m}$ papaverine was perfused to induce complete relaxation. Vasoconstrictions are expressed as the peak increase in mean perfusion pressure from the baseline value before PNS or the noradrenaline injection. Vasodilation is expressed as the percentage of maximum relaxation induced by the perfusion of papaverine.

\section{Immunohistochemical study}

Under pentobarbital-Na (50 $\mathrm{mg} \mathrm{kg}^{-1}$, intraperitoneally) anesthesia, the superior mesenteric artery of OLETF $(n=7)$ and LETO $(n=7)$ rats was cannulated with polyethylene tubing, and Zamboni solution ( $2 \%$ paraformaldehyde and $15 \%$ picric acid in $0.15 \mathrm{~m}$ phosphate buffer) was infused. Then, the mesenteric artery was removed with the intestines as described previously. ${ }^{24,25}$ The third branch of the mesenteric artery proximal to the intestine was removed and immersion fixed in Zamboni solution for $48 \mathrm{~h}$. After fixation, the artery was repeatedly rinsed in phosphate-buffered saline (PBS), immersed in PBS containing $0.5 \%$ Triton X-100 overnight and incubated with PBS containing normal goat serum (1:100) for $60 \mathrm{~min}$. The tissue was then incubated with a rabbit polyclonal anti-CGRP (Biogenesis, Oxford, UK) antiserum at a dilution of 1:300 or a rabbit polyclonal anti-NPY (Phoenix Pharmaceuticals, Belmont, CA, USA) antiserum at $1: 300$ for $72 \mathrm{~h}$ at $4{ }^{\circ} \mathrm{C}$. After the incubation, the artery was washed in PBS, and the sites of antigen-antibody reactivity were detected 
by incubation with fluorescein-5-isothiocyanate-labeled goat anti-rabbit IgG (1:100) (ICN Pharmaceuticals, Aurora, OH, USA) for $60 \mathrm{~min}$. Thereafter, the artery was thoroughly washed in PBS, mounted on a slide, coverslipped with glycerol/PBS $\left(2: 1\left(\mathrm{vv}^{-1}\right)\right)$, and observed under a confocal laser scanning microscope (CLSM510; Carl Zeiss, Tokyo, Japan) in the Okayama University Medical School Central Research Laboratory.

\section{Immunohistochemical analysis}

The immunostaining density of CGRP-like immunoreactive (CGRP-LI) and NPY-like immunoreactive (NPY-LI) nerve fibers was analyzed using the method described by Hobara et al. ${ }^{24,25}$ Because the fluorescence intensity differed depending on the day of the experiment, the mesenteric arteries from OLETF and LETO rats were isolated, fixed and immunostained at the same time on the same day and mounted on the same glass slide. For the quantitative evaluation of CGRP-LI and NPY-LI, confocal projection images of CGRP and NPY immunostaining, which were patched together with 8-10 overlapping images $(0.1 \mu \mathrm{m}$ scanning $)$, were magnified $\times 20$ and digitized as TIF images using a digital camera system (Olympus SP-1000; Olympus, Tokyo, Japan) and imported into a computer running Windows XP (Toshiba, Tokyo, Japan). The stored digital images were analyzed using image-processing software (Simple PCI; Compix, Imaging Systems, Cranberry Township, PA, USA). The extraction of specific color and measured field commands were used to extract the CGRP-LI and NPY-LI areas, which were stained green. Extraction of the signal was performed using specific protocols based on the hue, lightness and saturation color parameters. A measured field of $100 \mu \mathrm{m} \times 100 \mu \mathrm{m}\left(10000 \mu \mathrm{m}^{2}\right)$ that contained the adventitial layer including immunostained perivascular nerve fibers was randomly selected in magnified images of the whole-mount artery. An objective areas' command was used to calculate the percentages of CGRP-LI- and NPY-LI-positive areas. The intensity of staining was estimated using a point-counting computer program, and the background level was subtracted from the experimental value to yield the corrected intensity. The average density of three arteries was taken as the nerve density per animal.

To determine the number of CGRP-LI and NPY-LI fibers, five horizontal lines were drawn on the image of the blood vessel in the same region where the density was estimated by computer analysis. Then, the number of fibers that crossed each line was counted, and the average number in the three arteries was taken as the total number of fibers per animal. Because immunostaining of NPY-LI and CGRP-LI fibers in some preparations showed weak fluorescent staining because of weak antibody absorption, five of seven results were selected.

\section{Statistical analysis}

The experimental results are presented as the mean \pm s.e.m. A one-way analysis of variance followed by Tukey's test or a two-way analysis of variance was used to determine statistical significance, when appropriate. The correlation analysis was performed using Pearson's correlation test. A value of $P<0.05$ was considered statistically significant.

\section{RESULTS}

Changes in body weight and food and fluid intakes

As shown in Table 1, body weight in OLETF rats at 8 and 25 weeks of age was significantly greater than that in age-matched LETO rats. OLETF rats showed a decrease in total food and fluid intake, but a significant difference was only found between the strains at 8 weeks of age.

Changes in blood glucose levels, the glucose-insulin index, plasma insulin levels and total cholesterol and triglyceride levels

As shown in Table 1, the plasma glucose levels in OLETF rats at age 25 weeks, but not 8 weeks, were significantly greater than those in LETO rats. OLETF rats at 8 and 25 weeks of age showed two- and eightfold greater plasma insulin levels than the LETO rats, respectively. In 25week-old OLETF rats, the HOMA-IR (indicating the extent of insulin resistance) was 10 -fold greater than that in age-matched LETO rats.
Table 1 Changes in parameters in OLETF and LETO rats at 8 and 25 weeks of age

\begin{tabular}{lll}
\hline & 8 weeks old & 25 weeks old \\
\hline Body weight $(g)$ & & \\
LETO & $244.7 \pm 5.0$ & $498.8 \pm 4.8$ \\
OLETF & $282.0 \pm 4.0^{* *}$ & $624.6 \pm 7.5^{* *}$
\end{tabular}

Total food intake (g per week)

$\begin{array}{lll}\text { LETO } & 263.0 \pm 28.6 & 250.8 \pm 2.5\end{array}$

$\begin{array}{lll}\text { OLETF } & 206.3 \pm 11.0 & 203.3 \pm 10.00\end{array}$

Total fluid intake (g per week)

$\begin{array}{lll}\text { LETO } & 166.3 \pm 6.5 & 172.5 \pm 9.1\end{array}$

OLETF $109.6 \pm 16.3^{*} \quad 144.2 \pm 7.5$

Blood glucose $\left(\mathrm{mgdl}^{-1}\right)$

$\begin{array}{lll}\text { LETO } & 104.0 \pm 2.9 & 138.0 \pm 4.9\end{array}$

$\begin{array}{ll}\text { OLETF } & 109.5 \pm 2.9 \\ \end{array}$

Plasma insulin $\left(\mu \cup \mathrm{ml}^{-1}\right)$

LETO $\quad 6.6 \pm 0.7 \quad 11.4 \pm 2.2$

$\begin{array}{ll}\text { OLETF } & 11.7 \pm 2.1^{*} \\ \end{array}$

HOMA-IR

$\begin{array}{lll}\text { LETO } & 1.67 \pm 0.1 & 3.9 \pm 0.9\end{array}$

$\begin{array}{lll}\text { OLETF } & 3.1 \pm 0.5 & 44.8 \pm 4.6^{* *}\end{array}$

Plasma total cholesterol ( $\left.m g \mathrm{dl}^{-1}\right)$

LETO $\quad 77.8 \pm 0.5 \quad 117.1 \pm 1.4$

$\begin{array}{lll}\text { OLETF } & 84.5 \pm 4.7 & 123.4 \pm 10.9^{* *}\end{array}$

Plasma triglyceride $\left(\mathrm{mgdl}^{-1}\right)$

LETO $\quad 50.9 \pm 5.6 \quad 121.5 \pm 32.9$

$\begin{array}{lll}\text { OLETF } & 92.0 \pm 3.6^{* *} & 297.2 \pm 17.6^{* *}\end{array}$

Systolic blood pressure $(\mathrm{mm} \mathrm{Hg})$

$\begin{array}{lll}\text { LETO } & 120.6 \pm 1.7 & 135.4 \pm 1.5 \\ \text { OLETF } & 134.7 \pm 2.4^{* *} & 159.0 \pm 2.5^{* *}\end{array}$

Abbreviations: HOMA-IR, homeostasis model assessment ratio; LETO, Long-Evans Tokushima Otsuka rat; OLETF, Otsuka Long-Evans Tokushima Fatty.

Values are expressed as the mean \pm s.e.m. from five to seven rats.

$* P<0.05, * * P<0.01$ vs. age-matched LETO rats.

The plasma total cholesterol and triglyceride levels in 25-week-old but not 8 -week-old OLETF rats were significantly higher than those in age-matched LETO rats (Table 1 ).

\section{Changes in systolic blood pressure and heart rate}

As shown in Table 1, the systolic blood pressure in OLETF rats increased from 8 to 25 weeks of age, and significant differences were found between OLETF and LETO rats at 8 and 25 weeks of age. However, there was no significant difference in heart rate between OLETF rats and LETO rats during the observation period (data not shown).

\section{Changes in vasoconstrictor responses to PNS and injections of noradrenaline}

As shown in Figures $1 \mathrm{a}$ and b, PNS at 4, 8 and $12 \mathrm{~Hz}$ in the perfused mesenteric vascular beds of LETO and OLETF rats with resting tone produced a frequency-dependent increase in perfusion pressure because of vasoconstriction. The vasoconstriction was abolished by 
the $\alpha 1$-adrenoceptor antagonist prazosin and the adrenergic neuron blocker guanethidine (data not shown), indicating that the PNS response is mediated by noradrenaline released from the periarterial adrenergic nerves. In addition, injections of noradrenaline at 5 and $10 \mathrm{nmol}$ induced a concentration-dependent vasoconstriction (Figures 1c and d), which was blocked by prazosin but not guanethidine (data not shown), indicating that the noradrenaline response was mediated by the stimulation of postsynaptic $\alpha 1$-adrenoceptors.

As shown in Figure 1b, the PNS ( 8 and $12 \mathrm{~Hz}$ )-induced vasoconstrictions in OLETF rats at age 25 weeks, but not 8 weeks, were significantly greater than those in LETO rats.
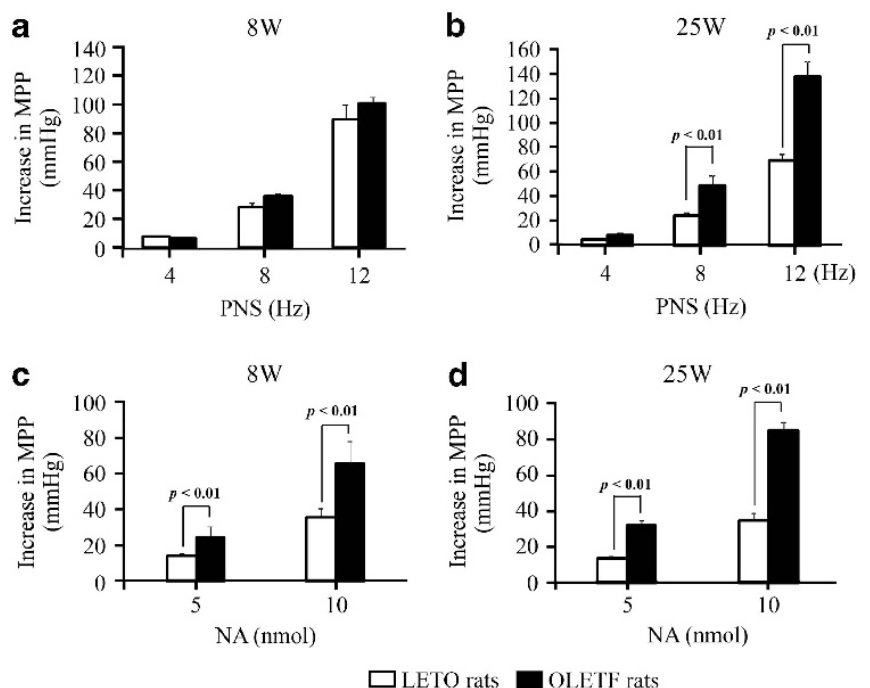

Figure 1 Vasoconstrictor responses to perivascular nerve stimulation (PNS) ( $\mathbf{a}$ and $\mathbf{b} ; 4-12 \mathrm{~Hz}$ ) and injections of noradrenaline (NA) (c and $\mathbf{d} ; 5$ and $10 \mathrm{nmol}$ ) in perfused mesenteric vascular beds isolated from Long-Evans Tokushima Otsuka rat (LETO) and Otsuka Long-Evans Tokushima Fatty (OLETF) rats at 8 ( $8 \mathrm{w}$; $\mathbf{a}$ and $\mathbf{c})$ and $25(25 \mathrm{w}$; b and $\mathbf{d})$ weeks of age. Open and closed bars indicate LETO rats and OLETF rats, respectively. Each bar indicates the mean \pm s.e.m. for four to five experiments. MPP, mean perfusion pressure.
As shown in Figures $1 \mathrm{c}$ and $\mathrm{d}$, the vasoconstrictor responses to noradrenaline injection at 5 and $10 \mathrm{nmol}$ in 8 - and 25-week-old OLETF rats were significantly greater than those in age-matched LETO rats.

Changes in the distribution of sympathetic NPY-LI nerve fibers in mesenteric arteries

As shown in Figures 2a and b, the distal small mesenteric arteries from LETO and OLETF rats had dense NPY-LI nerve fibers, which surrounded the arteries as in a network. As shown in Figures $2 \mathrm{c}$ and $\mathrm{d}$, the density and number of NPY-LI nerve fibers in 8 - and 25-weekold OLETF rats were similar to those in age-matched LETO rats.

There were significant positive correlations between the density and number of NPY-LI nerve fibers in LETO rats at $8\left(P<0.05, R^{2}=0.22\right)$ and $25\left(P<0.05, R^{2}=0.58\right)$ weeks of age and in OLETF rats at 8 $\left(P<0.05, R^{2}=0.35\right)$ and $25\left(P<0.01, R^{2}=0.70\right)$ weeks of age.

Changes in vasodilator responses to PNS and injection of CGRP In LETO and OLETF preparations with active tone produced by the continuous perfusion of $7 \mu \mathrm{M}$ methoxamine in the presence of $5 \mu \mathrm{M}$ guanethidine, PNS at 2, 4 and $8 \mathrm{~Hz}$ caused a frequency-dependent decrease in perfusion pressure owing to vasodilation (Figures $3 \mathrm{a}$ and b). The vasodilator response to PNS has been shown to be mediated by CGRPergic nerves because the response is blocked by a CGRP receptor antagonist (CGRP (8-37)) and a CGRP depletor (capsaicin)..$^{26,27}$ In addition, injections of CGRP at 25, 50 and $100 \mathrm{nmol}$ induced concentration-dependent vasodilation (Figures $3 \mathrm{c}$ and $\mathrm{d}$ ), which has been shown to be mediated by postsynaptic CGRP receptors. $^{28}$ There was no significant difference in the methoxamine-induced increases in mean perfusion pressure before the PNS and CGRP injections between LETO and OLETF rats (data not shown). As shown in Figure 3, nearly all of the PNS- and CGRP injection-induced vasodilator responses in 8- and 25-week-old OLETF rats were significantly smaller than those in age-matched LETO rats (Figures $3 \mathrm{a}$ and $\mathrm{b}$, respectively), although this was not true for PNS at $2 \mathrm{~Hz}$ in 8 -week-old rats or CGRP at $25 \mathrm{pmol}$ in 25-week-old rats.

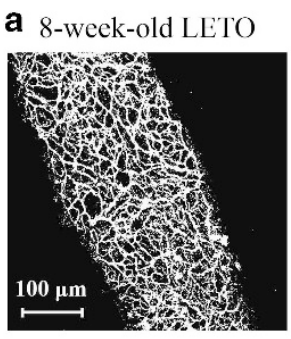

25-week-old LETO

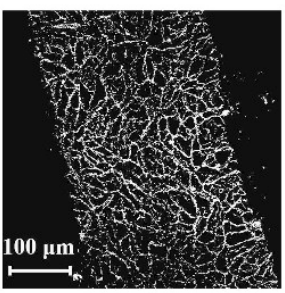

b 8-week-old OLETF

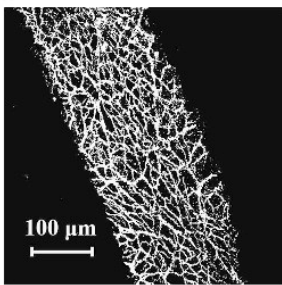

25-week-old OLETF

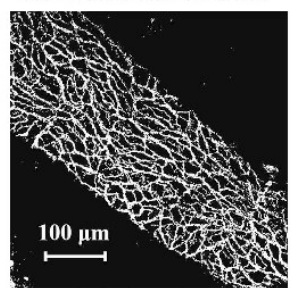

C
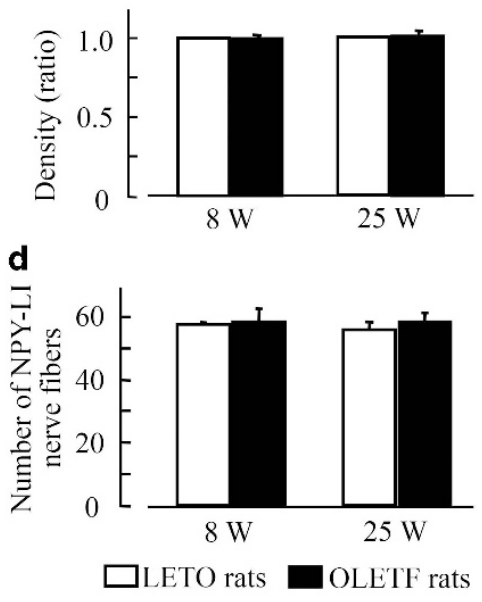

Figure 2 Typical images (a and $\mathbf{b}$ ) and bar graphs showing the density (c) and number (d) of neuropeptide Y (NPY)-like immunoreactivity (LI)-containing nerve fibers in small mesenteric arteries isolated from Long-Evans Tokushima Otsuka rat (LETO) and Otsuka Long-Evans Tokushima Fatty (OLETF) rats at 8 $(8 w)$ and $25(25 w)$ weeks of age. The scale bar $=100 \mu \mathrm{m}$ in the left lower corner of each image. Each bar indicates the mean \pm s.e.m. for five experiments. 
Changes in the distribution of CGRP-LI nerve fibers in mesenteric arteries

As shown in Figures $4 \mathrm{a}$ and $\mathrm{b}$, dense innervation of CGRP-LI nerve fibers was observed in the mesenteric arteries of LETO and OLETF rats at 8 and 25 weeks of age. CGRP-LI nerve fibers innervated the artery similar to sympathetic NPY-LI nerves but were less dense than a

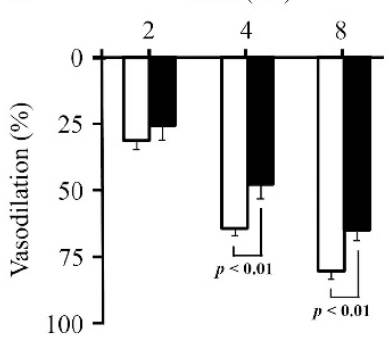

$8 \mathrm{~W}$

c

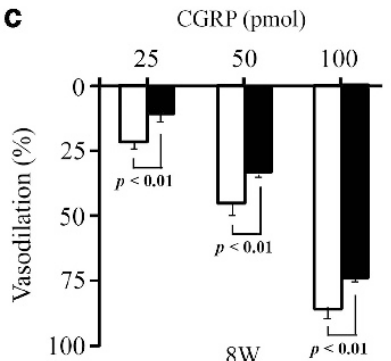

b

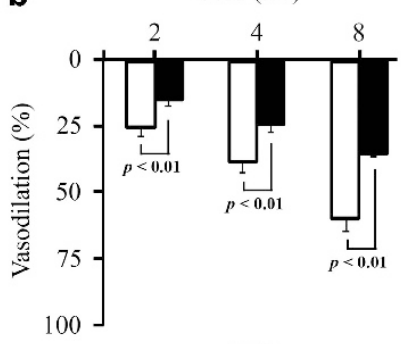

$25 \mathrm{~W}$

d

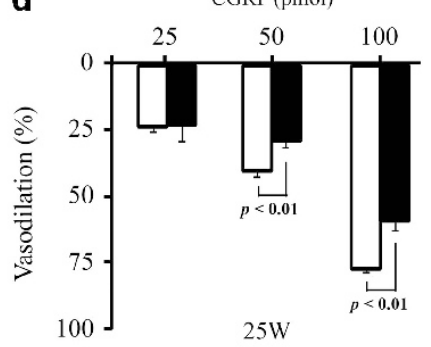

$\square$ LETO rats

OLETF rats

Figure 3 Vasodilator responses to perivascular nerve stimulation (PNS) (a and $\mathbf{b} ; 2-8 \mathrm{~Hz}$ ) and injections of calcitonin gene-related peptide (CGRP) (c and d; 25-100 pmol) in perfused mesenteric vascular beds isolated from Long-Evans Tokushima Otsuka rat (LETO) and Otsuka Long-Evans Tokushima Fatty (OLETF) rats at 8 ( 8 w; a and c) and 25 (25w; b and d) weeks of age. Open and closed bars indicate LETO rats and OLETF rats, respectively. Each bar indicates the mean \pm s.e.m. for four to five experiments.
NPY-LI nerves. The density and number of CGRP-LI nerve fibers in OLETF rats at 8 and 25 weeks of age were significantly smaller than those in age-matched LETO rats, as shown in Figures $4 \mathrm{c}$ and $\mathrm{d}$. In addition, the number of CGRP-LI nerve fibers in 25-week-old OLETF rats was significantly smaller than that in 8 -week-old OLETF rats (Figure 4d).

There were significant positive correlations between the density and number of CGRP-LI nerve fibers in LETO rats at $8 \quad(P<0.01$, $\left.R^{2}=0.58\right)$ and $25\left(P<0.01, R^{2}=0.25\right)$ weeks of age and in OLETF rats at $8\left(P<0.05, R^{2}=0.24\right)$ and $25\left(P<0.01, R^{2}=0.46\right)$ weeks of age.

Changes in vasodilator responses to injections of ACh and SNP At 8 and 25 weeks of age, in OLETF preparations with active tone produced by methoxamine $(7 \mu \mathrm{M})$ in the presence of guanethidine $(5 \mu \mathrm{M})$, the injection of $\mathrm{ACh}(10,50$ and $100 \mathrm{pmol})$ induced a concentration-dependent vasodilation that was endothelium dependent. The ACh-induced vasodilation in OLETF rats at 25 weeks old was significantly smaller than that in age-matched LETO rats (Figure 5b).

Injections of SNP at 100 and 1000 pmol into LETO and OLETF rat preparations induced a concentration-dependent vasodilation (Figures $5 \mathrm{c}$ and $\mathrm{d}$ ) that was endothelium independent. As shown in Figure 5d, the SNP (1000 pmol)-induced vasodilator responses in 25-week-old OLETF rats were significantly smaller than those in age-matched LETO rats.

There was no significant difference in the methoxamine-induced increases in mean perfusion pressure before the ACh and SNP injections between LETO and OLETF rats (data not shown).

\section{DISCUSSION}

The present study demonstrated that the density of CGRP-LI nerves decreased with aging in OLETF rats compared with genetically related LETO rats. In contrast, the density of adrenergic NPY-LI nerves was similar in the two strains. These findings suggest that aging has a role in the changes in CGRPergic nerve function in OLETF rats. This is supported by the present findings demonstrating that the CGRPergic
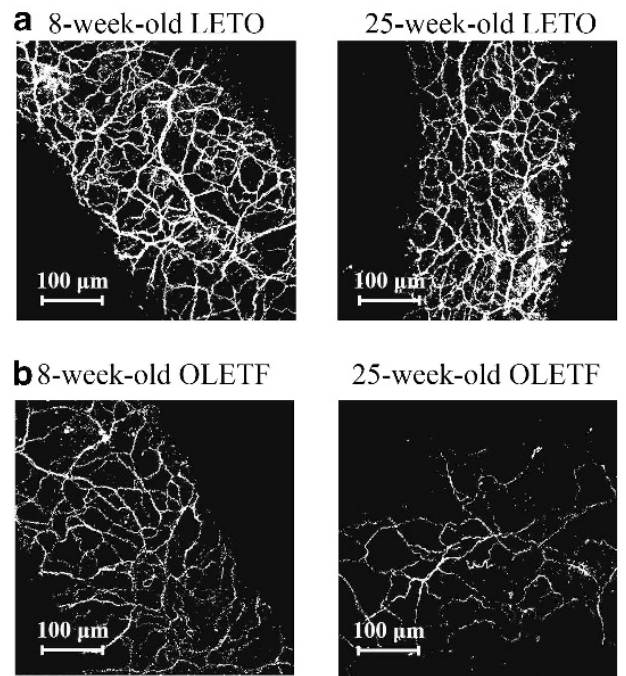
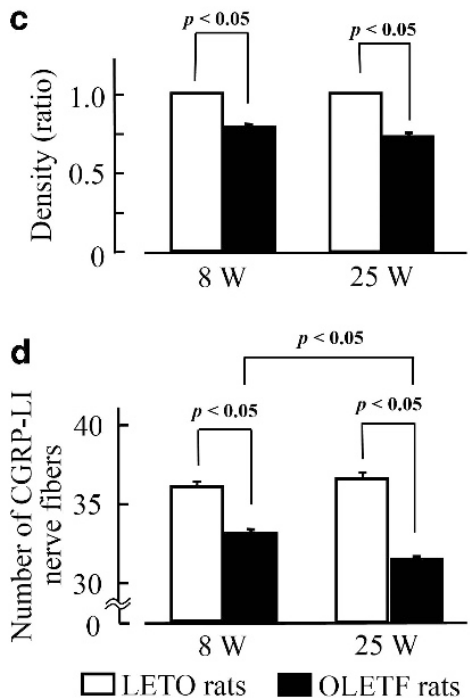

Figure 4 Typical images (a and $\mathbf{b}$ ) and bar graphs showing the density (c) and number (d) of calcitonin gene-related peptide (CGRP)-like immunoreactivity (LI)-containing nerve fibers in small mesenteric arteries isolated from Long-Evans Tokushima Otsuka rat (LETO) and Otsuka Long-Evans Tokushima Fatty (OLETF) rats at $8(8 \mathrm{w})$ and $25(25 \mathrm{w})$ weeks of age. The scale bar $=100 \mu \mathrm{m}$ in the left lower corner of each image. Each bar indicates the mean \pm s.e.m. for five experiments. 
a

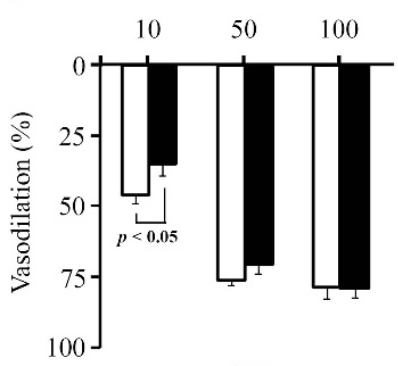

$8 \mathrm{~W}$

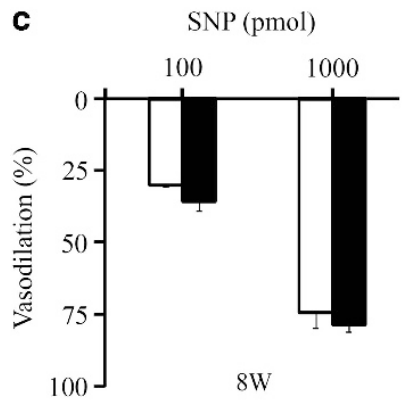

b

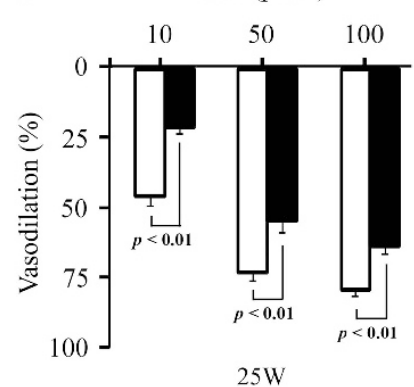

d

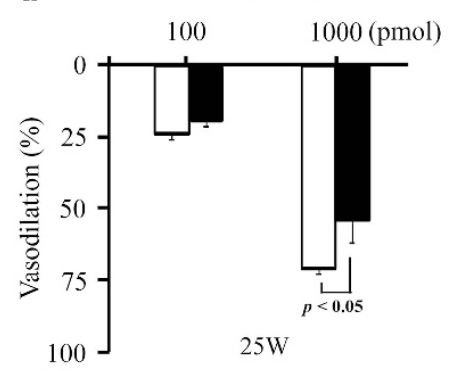

$\square$ LETO rats OLETF rats

Figure 5 Bar graphs showing vasodilator responses to injections of acetylcholine (ACh) (a and $\mathbf{b} ; 10-100 \mathrm{pmol}$ ) and sodium nitroprusside (SNP) (c and d; 100 and $1000 \mathrm{pmol}$ ) in perfused mesenteric vascular beds isolated from Long-Evans Tokushima Otsuka rat (LETO) and Otsuka LongEvans Tokushima Fatty (OLETF) rats at $8(8 \mathrm{w})$ and $25(25 \mathrm{w})$ weeks of age. Open and closed bars indicate LETO rats and OLETF rats, respectively. Each bar indicates the mean \pm s.e.m. for four to five experiments.

nerve-mediated vasodilator response to PNS was significantly smaller in mesenteric vascular beds from OLETF rats than in the preparations from age-matched LETO rats and that it was age-related. Our previous study showed that CGRPergic vasodilator nerves functionally attenuated adrenergic nerve-mediated vasoconstriction in vitro using rat mesenteric resistance arteries, suggesting that adrenergic and CGRPergic nerves regulate the tone of resistance arteries through reciprocal interactions. ${ }^{29}$ Therefore, it is assumed that decreased CGRPergic nerve function results in increased adrenergic nerve function in OLETF rats. This is also supported by the present findings, which demonstrated that the adrenergic nerve-mediated vasoconstrictor response to PNS in OLETF preparations increased, although the density of adrenergic NPY-LY nerves was unchanged.

Several human studies have revealed that changes in sympathetic neuronal control of the circulation have a major effect on age-related hypertension. ${ }^{16,17}$ Surprisingly, the present findings demonstrated that the extent of adrenergic NPY-LI innervation in the mesenteric arteries of OLETF rats at 8 and 25 weeks did not change with aging. Thus, it appears that augmented adrenergic nerve-mediated vasoconstriction results primarily from a significant decrease in the density and number of CGRPergic nerves, and not from changes in the density of sympathetic adrenergic nerves.

Our recent report showed that FDRs, which had insulin resistance, hyperinsulinemia and hypertension, exhibited decreases in the density of CGRP-LI nerve fibers innervating the mesenteric arteries and the levels of CGRP in the dorsal root ganglia, a prominent site of CGRP synthesis, suggesting that decreased CGRPergic function is involved in the development of hypertension in FDRs. ${ }^{30}$

Our suggestion of a possible relationship between hypertension and insulin resistance is supported by clinical evidence indicating that patients with type 2 diabetes mellitus have a higher risk of developing hypertension. ${ }^{3-5}$ Furthermore, our previous report demonstrated that chronic hyperinsulinemia and insulin resistance in FDRs resulted in augmented adrenergic nerve-mediated vasoconstriction and reduced CGRPergic nerve-mediated vasodilation and that treatment of FDRs with pioglitazone, an insulin-sensitizing agent, ameliorated the impaired functioning of the neuronal vascular control system resulting from chronic hyperinsulinemia. ${ }^{13}$ Therefore, it seems likely that a clear association exists between the development of hypertension and reduced CGRPergic nerve-mediated vasodilation resulting from the decreased density of CGRPergic nerve fibers in the mesenteric arteries of OLETF rats with insulin resistance and/or hyperinsulinemia.

CGRP synthesis and the expression of both $\alpha$ - and $\beta$-CGRP mRNA in the dorsal root ganglia have been shown to be decreased in spontaneously hypertensive rats. ${ }^{31}$ Moreover, treatment of hypertension with CGRP attenuates pressure to protect against organ damage. ${ }^{32}$ These findings suggest that CGRP is a key factor regulating the total peripheral resistance of the systemic circulation. ${ }^{33}$

In a previous in vitro study, insulin was shown to act on CGRP receptors and cause vasodilatation in rat mesenteric resistance arteries. ${ }^{34}$ In addition, CGRPergic nerve terminals have presynaptic CGRP receptors that inhibit neurotransmitter CGRP release via a negative feedback mechanism. ${ }^{35}$ Therefore, it seems possible that acute or chronic increases in the plasma insulin level stimulate presynaptic CGRP receptors to inhibit neurotransmitter CGRP release from CGRPergic nerve terminals, which decreases the vasodilator response to PNS in OLETF rats. However, further studies will be needed to clarify whether insulin activates presynaptic CGRP receptors in CGRPergic nerves.

In the present study, ACh-induced vasodilation, which is endothelium dependent, was strongly suppressed in the mesenteric arteries of OLETF rats at 8 and 25 weeks of age when compared with agematched LETO rats, suggesting that chronic hyperinsulinemia results in endothelial dysfunction. The present results are in accordance with clinical findings ${ }^{36-38}$ showing endothelial dysfunction in patients with type 2 diabetes, in which insulin resistance has a central role in the observed dysfunction. Furthermore, the present study shows that noradrenaline-induced vasoconstriction, which is mediated by postsynaptic $\alpha$-adrenoceptors on blood vessels, is markedly greater in the mesenteric arteries of OLETF rats than in those of LETO rats. Because the endothelium counteracts vasoconstriction by releasing endothelium-derived relaxing factors, ${ }^{39}$ it seems likely that the augmented noradrenaline-induced vasoconstriction in OLETF rats is due partly to reduced endothelial function. Thus, the change in vascular responsiveness appears to affect the vasoconstriction that occurs in response to noradrenaline. Moreover, the SNP-induced vasodilator response, which is endothelium independent, was also suppressed in the mesenteric arteries of 25-week-old OLETF rats. These findings also suggest that the vasoactivity of vascular smooth muscle, including the decreased transformation to NO, decreases with aging in the mesenteric arteries of OLETF rats.

In conclusion, the present findings suggest that insulin resistance and/or hyperinsulinemia in OLETF rats induces enhanced adrenergic nerve-mediated vasoconstriction and reductions in CGRPergic nervemediated vasodilation and the extent of CGRPergic innervation in mesenteric vascular beds and that these phenomena are partially age related. It is likely that the disruption of the neuronal vascular control system resulting from decreased innervation of perivascular 
CGRPergic nerves induced by insulin resistance and/or hyperinsulinemia in OLETF rats may be responsible for the development of hypertension that is insulin resistant.

\section{CONFLICT OF INTEREST}

The authors declare no conflict of interest.

\section{ACKNOWLEDGEMENTS}

We express our gratitude to Otsuka Pharmaceutical for providing the LETO and OLETF rats.

1 Adler AI, Stratton IM, Neil HA, Yudkin JS, Matthews DR, Cull CA, Wrigh AD, Tumer RC, Holman RR. Association of systolic blood pressure with macrovascular and microvascular complications of type 2 diabetes (UKPDS 36): prospective observational study. BMJ 2000; 321: 412-419.

2 Norhammar A, Tenerz A, Nilsson G, Hamsten A, Efendíc S, Rydén L, Malmberg K. Glucose metabolism in patients with acute myocardial infarction and no previous diagnosis of diabetes mellitus: a prospective study. Lancet 2002; 359: 2140-2144.

3 Skarfors ET, Selinus KI, Lithell HO. Risk factors for developing non-insulin dependent diabetes: a 10 year follow up of men in Uppsala. BMJ 1991; 303: 755-760.

4 Haffner SM, Valdez RA, Hazuda HP, Mitchell BD, Morales PA, Stern MP. Prospective analysis of the insulin-resistance syndrome (syndrome X). Diabetes 1992; 41: 715-722.

5 Lissner L, Bengtsson C, Lapidus L, Kristjansson K, Wedel H. Fasting insulin in relation to subsequent blood pressure changes and hypertension in women. Hypertension 1992; 20: 797-801.

6 Meehan WP, Buchanan TA, Hsueh W. Chronic insulin administration elevates blood pressure in rats. Hypertension 1994: 23: 1012-1017.

7 Takatori S, Mizote M, Zamami Y, Kurosaki Y, Kawasaki H. Effects of insulin on vascular responses to spinal cord stimulation and vasoactive agents in pithed rats. $\mathrm{Br} J$ Pharmacol 2003; 140: 1137-1145.

8 DeFronzo RA. Is insulin resistance atherogenic? Possible mechanisms. Atherosclerosis 2006; 7: 11-15.

9 Kashyap SR, DeFronzo RA. The insulin resistance syndrome: physiological considerration. Diab Vasc Dis Res 2007; 4: 13-19.

10 Grundy SM, Becker D, Clark LT, Cooper RS, Denke MA, Howard WJ, Hunninghake DB, Illingworth DR, Luepker RV, McBride P, McKenney JM, Pasternak RC, Stone NJ, Horn LV. Third report of the National Cholesterol Education Program (NCEP). Expert panel on detection, evaluation and treatment of high blood cholesterol in adults (Adult Treatment Panel III) final report. Circulation 2002; 106: 3143-3421.

11 Miranda PJ, Defronzo RA, Califf RM, Guyton JR. Metabolic syndrome: evaluation of pathologic and therapeutic outcome. Am Heart J 2005; 149: 20-32.

12 Takatori S, Zamami Y, Mio M, Kurosaki Y, Kawasaki H. Chronic hyperinsulinemia enhances adrenergic vasoconstriction and decreases calcitonin gene-related peptide-containing nerve-mediated vasodilation in pithed rats. Hypertens Res 2006; 29: 361-368.

13 Takatori S, Zamami Y, Yabumae N, Hanafusa N, Mio M, Egawa T, Kawasaki H. Pioglitazone opposes neurogenic vascular dysfunction associated with chronic hyperinsulinaemia. Br J Pharmacol 2008; 153: 1388-1398.

14 Jin X, Amitani K, Zamami Y, Takatori S, Hobara N, Kawamura N, Hirata T, Wada A Kitamura Y, Kawasaki H. Ameliorative effect of Eucommia ulmoides Oliv. leaves extract (ELE) on insulin resistance and abnormal perivascular innervation in fructose-drinking rats. J Ethnopharmacol 2010; 128: 672-678.

15 Osborn JW, Fink GD, Sved AF, Toney GM, Raizada MK. Circulating angiotensin II and dietary salt: converging signals for neurogenic hypertension. Curr Hypertens Rep 2007 9: 228-235.

16 Sundlöf G, Wallin BG. Human muscle nerve sympathetic activity at rest. Relationship to blood pressure and age. J Physiol 1978; 274: 621-637

17 Narkiewicz K, Phillips BG, Kato M, Hering D, Bieniaszewski L, Somers VK. Genderselective interaction between aging, blood pressure, and sympathetic nerve activity. Hypertension 2005; 45: 522-525.

18 Kawasaki H, Saito A, Takasaki K. Age-related decrease of calcitonin gene-related peptide-containing vasodilator innervation in the mesenteric resistance vessel of the spontaneously hypertensive rat. Circ Res 1990; 67: 733-743.
19 Kawasaki $\mathrm{H}$. Effects of chronic administration of antihypertensive drugs on vasodilation mediated by calcitonin gene-related peptide-containing vasodilator nerves in spontaneously hypertensive rats. Clin Exp Pharmacol Physiol 1992; 19: 569-573.

20 Kawasaki H, Nuki Y, Yamaga N, Kurosaki Y, Taguchi T. Decreased depressor response mediated by calcitonin gene-related peptide (CGRP)-containing vasodilator nerves to spinal cord stimulation and levels of CGRP mRNA of the dorsal root ganglia in spontaneously hypertensive rats. Hypertens Res 2000; 23: 693-699.

21 Hobara N, Gessei-Tsutsumi N, Goda M, Takayama F, Akiyama S, Kurosaki Y, Kawasaki H. Long-term inhibition of angiotensin prevents reduction of periarterial innervation of calcitonin gene-related peptide (CGRP)-containing nerves in spontaneously hypertensive rats. Hypertens Res 2005; 28: 465-474.

22 Kawano K, Hirashima T, Mori S, Saitoh Y, Kurosumi M, Natori T. Spontaneous longterm hyperglycemic rat with diabetic complications. Otsuka Long-Evans Tokushima Fatty (OLETF) strain. Diabetes 1992; 41: 1422-1428.

23 Kawasaki H, Takasaki K, Saito A, Goto K. Calcitonin gene-related peptide acts as a novel vasodilator neurotransmitter in mesenteric resistance vessels of the rat. Nature 1988; 335: 164-167.

24 Hobara N, Goda M, Takayama F, Kawasaki H. Innervation and functional changes in mesenteric perivascular calcitonin gene-related peptide (CGRP)- and neuropeptide $Y$ NPY)-containing nerves followi topical phenol treatment. Neuroscience 2006; 141 1087-1099.

25 Hobara N, Goda M, Yoshida N, Takatori S, Kitamura Y, Mio M, Kawasaki H. Angiotensin II type 2 receptors facilitate reinnervation of phenol-lesioned vascular calcitonin generelated peptide-containing nerves in rat mesenteric arteries. Neuroscience 2007; 150: 730-741.

26 Han SP, Naes L, Westfall TC. Inhibition of periarterial nerve stimulation-induced vasodilation of the mesenteric arterial bed by CGRP (8-37) and CGRP receptor desensitization. Biochem Biophys Res Commun 1990; 168: 786-791.

27 Kawasaki H, Nuki C, Saito A, Takasaki K. NPY modulates neurotransmission of CGRPcontaining vasodilator nerves in rat mesenteric arteries. Am J Physiol 1991; 261 H683-H690.

28 Nuki C, Kawasaki H, Takasaki K, Wada A. Pharmacological characterization of presynaptic calcitonin gene-related peptide (CGRP) receptors on CGRP-containing vasodilator nerves in rat mesenteric resistance vessels. J Pharmacol Exp Ther 1994, 268: 59-64.

29 Kawasaki H, Nuki C, Saito A, Takasaki K. Role of calcitonin gene-related peptidecontaining nerves in the vascular adrenergic neurotransmission. J Pharmacol Exp Ther 1990; 252: 403-409.

30 Zamami Y, Takatori S, Hobara N, Yabumae N, Tangsucharit P, Jin X, Hashikawa H, Kitamura Y, Sasaki K, Kawasaki H. Hyperinsulinemia induces hypertension associated with neurogenic vascular dysfunction resulting from abnormal perivascular innervations in rat mesenteric resistance arteries. Hypertens Res 2011; 34: 1190-1196.

31 Li D, Peng J, Xin HY, Luo D, Zhang YS, Zhou Z, Jiang DJ, Deng HW, Li YJ. Calcitonin gene-related peptide-mediated antihypertensive and anti-platelet effects by rutaecarpine in spontaneously hypertensive rats. Peptide 2008; 29: 1781-1788.

32 Wang L, Wang DH. TRPV1 gene knockout impairs postischemic recovery in isolated perfused heart in mice. Circulation 2005; 112: 3617-3623.

33 Vaishnava P, Wang DH. Capsaicin sensitive-sensory nerves and blood pressure regulation. Curr Med Chem Cardiovasc Hematol Agents 2003; 1: 177-188.

34 Mimaki Y, Kawasaki H, Okazaki M, Nakatsuma A, Araki H, Gomita Y. Involvement of calcitonin gene-related peptide (CGRP) receptors in insulin-induced vasodilatation in mesenteric resistance blood vessels of rats. Br J Pharmacol 1998; 123: 1684-1690.

35 Nuki C, Kawasaki H, Takasaki K, Wada A. Pharmacological characterization of presynaptic calcitonin gene-related peptide (CGRP) receptors on CGRP-containing vasodilator nerves in rat mesenteric resistance vessels. J Pharmacol Exp Ther 1994; 268: 59-64.

36 Meeking DR, Cummings MH, Thorne S, Donald A, Clarkson P, Crook JR, Watts GF, Shaw KM. Endothelial dysfunction in type 2 diabetic subjects with and without microalbuminuria. Diabet Med 1999; 16: 841-847.

$37 \mathrm{Yu} \mathrm{HI}$, Sheu WH, Lai CJ, Lee WJ, Chen YT. Endothelial dysfunction in type 2 diabetes mellitus subjects with peripheral artery disease. Int J Cardiol 2001; 78: 19-25.

38 Green DJ, Maiorana AJ, Tschakovsky ME, Pyke KE, Weisbrod CJ, O'Driscoll G. Relationship between changes in brachial artery flow-mediated dilation and basal release of nitric oxide in subjects with type 2 diabetes. Am J Physiol Heart Circ Physiol 2006; 291: H1193-H1199.

39 Jin $X$, Satoh-Otonashi $Y$, Zamami $Y$, Koyama $T$, Sun $P$, Kitamura $Y$, Kawasaki $H$. Characterization of the inhibitory effect of vascular endothelium on agonist-induced vasoconstriction in rat mesenteric resistance arteries. J Pharmacol Sci 2008; 108 95-103. 